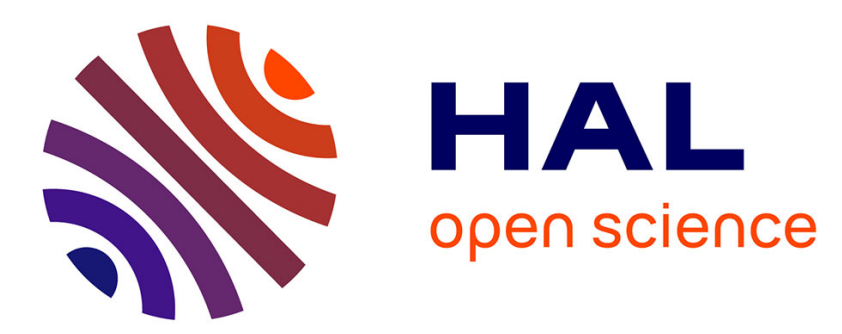

\title{
Modélisation du cadre spatio-temporel d'une caméra à balayage de fente
}

S. Verdelet, M. Gibert, G. Delaunay

\section{To cite this version:}

S. Verdelet, M. Gibert, G. Delaunay. Modélisation du cadre spatio-temporel d'une caméra à balayage de fente. Journal de Physique III, 1997, 7 (6), pp.1261-1275. 10.1051/jp3:1997187 . jpa-00249646

\section{HAL Id: jpa-00249646 https://hal.science/jpa-00249646}

Submitted on 1 Jan 1997

HAL is a multi-disciplinary open access archive for the deposit and dissemination of scientific research documents, whether they are published or not. The documents may come from teaching and research institutions in France or abroad, or from public or private research centers.
L'archive ouverte pluridisciplinaire HAL, est destinée au dépôt et à la diffusion de documents scientifiques de niveau recherche, publiés ou non, émanant des établissements d'enseignement et de recherche français ou étrangers, des laboratoires publics ou privés. 


\title{
Modélisation du cadre spatio-temporel d'une caméra à balayage de fente
}

\author{
S. Verdelet $\left({ }^{1,2, *}\right)$, M. Gibert $\left({ }^{1}\right)$ et G. Delaunay $\left({ }^{2}\right)$ \\ ( ${ }^{1}$ ) Commıssariat à l'Énergıe Atomique, Centre de Vaujours-Moronvilliers, \\ 51490 Pontfaverger, France \\ $\left({ }^{2}\right)$ Laboratoire d'Applications de la Micro-électronıque, Universıté de Reims, \\ Moulin de la Housse, BP 1039, 51687 Reims Cedex 2, France
}

(Reçu le 24 julllet 1996, révisé le 17 février 1997, accepté le 10 mars 1997)

PACS.02.60.x - Numerical approximation and analysis
PACS.06.60 Jn - High speed techniques
PACS.85.60.Me - Other optoelectronics device

Résumé. - Cet article porte sur l'élaboration d'un modèle géométrique du cadre spatiotemporel d'une caméra à balayage de fente. Cette dernière sert de chronomètre dans les expériences de détonique. Pour déterminer le modèle du cadre spatio-temporel, 1 l est nécessaire de déterminer les axes physiques du plan caméra à partır des axes images, grâce à l'emploi d'algorithmes du type moindres carrés récursıfs avec facteur d'oubli La détermination des paramètres du modèle va permettre d'améliorer l'exactitude des mesures chronométriques effectuées, tout en assurant un suivi temporel des performances des caméras, pour détecter d'éventuels défauts, susceptibles d'apparaitre au cours de l'expérience

\begin{abstract}
This artıcle deals with the elaboration of a geometric spatial and temporal model of a streak camera. This camera is used as a chronometer in the detonating experiments. To calculate the spatial and temporal geometric model, it is necessary to establish the axis of the plane face of the camera with axis recorded on a film. To achieve this, we use RLS algorithm with forgetting factor. The determination of parameters of model will allow to improve the accuracy of chronometric measures which we have carried out, by permitting a temporal steady of performances of streak cameras to detect possible defects during the experiment.
\end{abstract}

\section{Introduction}

Les caméras à balayage de fente sont couramment utilisées pour observer des phénomènes rapides, comme en détonique, où il s'agit d'étudier le comportement des composés explosifs. Les moyens expérimentaux mis en œuvre dans ce cadre doivent tenir compte de deux propriétés propres aux phénomènes physiques étudiés : leur nature transitoire et leur caractère destructif. De fait, la durée totale d'une expérience excède rarement quelques dizaines de microsecondes, les signaux recueillis au niveau des montages doivent être transmis en temps réel pour être enregistrés, ce qui explique que les moyens de mesure optoélectroniques occupent une place

$\left(^{*}\right)$ Auteur auquel doit être adressée la correspondance 
de choix. En effet, ils permettent de transporter aisément les signaux recueillis (sur plusieurs centaines de mètres) et d'atteindre des résolutions temporelles élevées.

De plus, le caractère destructeur des expériences de détonique pose le problème de la reproductibilité de la mesure, telle qu'elle est mise en œuvre classiquement, pour déterminer les propriétés statistiques du mesurande. Or, chaque expérience est unique du fait de sa configuration. Les expériences de détonique sont donc "monocoup". Il faut donc évaluer, voire corriger les défauts inhérents à l'instrumentation, pour s'assurer de la qualité dans les résultats de mesure, d'où l'intérêt de cette étude.

Pour cela, on se propose d'étudier le système d'enregistrement de la chaîne de chronométrie optoélectronique . la caméra à balayage de fente et son film associé. Le cadre spatio-temporel généré par la caméra va être modélisé, de façon à définir un jeu de paramètres caractérisant le fonctionnement dynamique de celle-ci, tout en améliorant l'exactitude des mesures.

Remarquons que d'autres techniques ont déjà été proposées pour modéliser les erreurs de linéarité liées au fonctionnement de caméras enregistrant des signaux transitoires rapides [1] ou les distorsions statiques d'un système de balayage couplé à un CCD [2]. Mais ces divers travaux ne permettent pas d'obtenir un modèle des déformations géométriques du cadre spatio-temporel généré par la caméra en fonctionnement dynamique, ce modèle étant lié aux caractéristiques physiques de la caméra, et ceci indépendamment de la durée de balayage sélectionnée.

\section{Principe de fonctionnement et d'exploitation des caméras électroniques à balayage de fente}

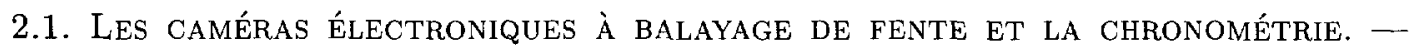
Différents paramètres sont nécessaires pour résoudre les problèmes de détonique. S'agissant des paramètres cinématiques, les techniques utilisées concernent principalement l'observation spatiale des phénomènes en fonction du temps, ainsi que leur chronométrie.

Lors d'une expérience, le nombre de signaux chronométrés peut nécessiter plusieurs centaines de voies de mesure. Pour des raisons d'encombrement, de coût mais aussi de précision, des capteurs à fibre optique ont été mis en œuvre avec les caméras à balayage de fente pour réaliser la chronométrie des déplacements d'interfaces se produisant lors de l'expérimentation d'un édifice pyrotechnique [3]. La figure 1 représente le dispositif expérimental de la chaîne de chronométrie.

Le capteur émet un sıgnal lumineux proportıonnel à un gradient de pression (choc). Le transport du signal issu de chaque sonde est assuré par une ligne de transmission optique, dont la longueur varie de quelques dizaines à quelques centaines de mètres Ces fibres optiques sont alignées de manière à constituer une fente sur l'entrée du tube convertisseur d'images d'une caméra à balayage de fente. Deux types de caméras électroniques à balayage de fente sont utilisées : TSN 503 et TSN 506, de la société Thomson C.S.F. [4], dont la résolution dynamique est de $16 \mathrm{pl} \mathrm{mm^{-1 }}$ (1 paire de ligne représente environ $62,5 \mu \mathrm{m}$ sur le film). Grâce au dispositif adaptateur (nez optique) placé devant la caméra, cette dernière dispose d'une capacité d'enregistrement de 54 voies de mesure.

Pour déterminer la chronométrie des signaux enregistrés sur un film à l'aide d'une caméra, il est nécessaire de disposer d'une base de temps [5]. À cet effet, un générateur d'impulsions (TSN 550, de la société Thomson C.S.F.) délivre des séries d'impulsions lumineuses périodiques. Typiquement, chaque film dispose de deux bases de temps synchrones encadrant l'ensemble des signaux. La chronométrie des impulsions lumineuses est connue, grâce au signaux électriques S1 et S10 enregistrés sur un chronomètre électronique. Ces signaux fournissent respectivement, le temps de la première et de la dixième impulsion lumineuse. Deux autres signaux électriques 


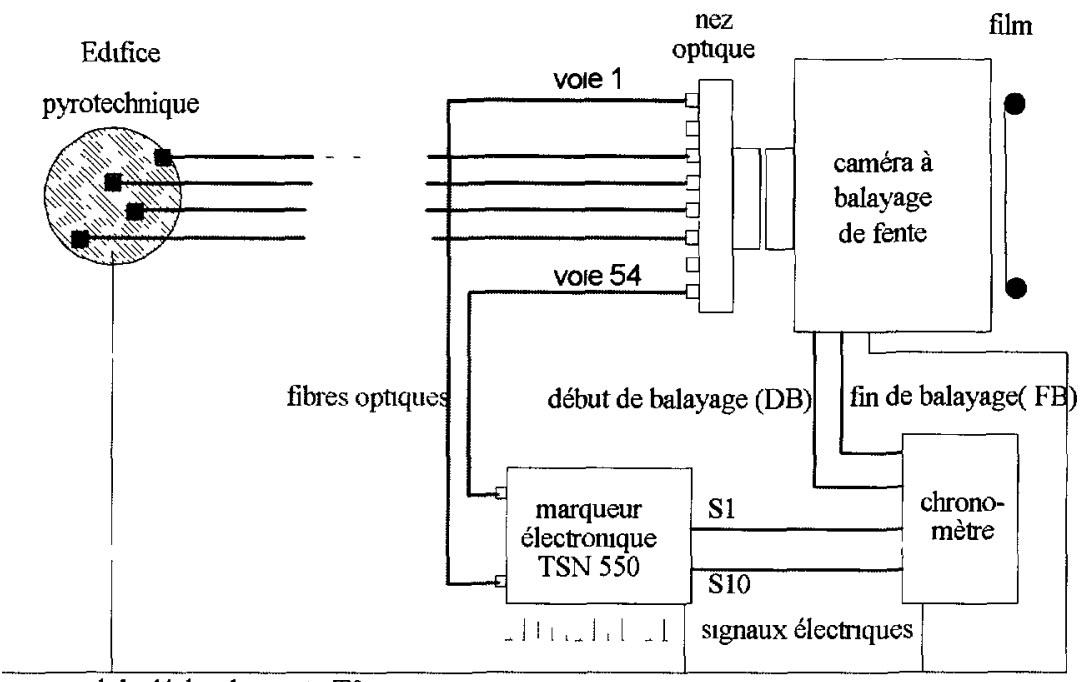

signal de déclenchement T0

Fig. 1. - La chaîne de chronométrie optoélectronique.

[Principle of the experimental optoelectronic and temporal calibration arrangement.]

sont enregistrés par ce chronomètre : ce sont les signaux émis par la caméra pour indiquer le début et la fin de balayage.

Chaque caméra utilisée dans la chaîne de chronométrie optoélectronique enregistre en même temps et en continu les signaux et les deux bases temporelles sur un support photographique (film). Ce dernier est développé puis, analysé en temps différé dans un laboratoire de traitement d'images, pour obtenir des cartes chronométriques des déplacements d'interfaces. La figure 2 représente un tel film obtenu lors d'une expérimentation. Le film utilisé avec la caméra est au format $5 \times 4$ pouces. La surface utile sur ce film correspond à la taille de l'image en sortie de la caméra, c'est-à-dire $50 \mathrm{~mm}$ pour la hauteur et $25 \mathrm{~mm}$ pour la largeur.

L'ensemble de l'instrumentation mis en œuvre pour l'étude d'un édifice pyrotechnique est synchronisé avec les événements à observer au moyen d'un signal de déclenchement. Typiquement, les durées de balayage (donc d'enregistrement), utilisées lors des expériences de détonique, s'étalent de 1 à $20 \mu \mathrm{s}$. Sur les 54 entrées du nez adaptateur de la caméra, les voies 1 et 54 , placées aux deux extrémités de la fente, sont utilisées pour la base de temps, les voies 3 à 52 reçoivent les signaux à enregistrer.

2.2. Principe de fonctionnement. - L'élément principal de la caméra est le tube convertisseur d'images (Fig. 3). À l'aide du nez adaptateur, les fibres des voies de mesures sont alignées devant la photocathode. Celle-ci convertit les photons en électrons qui sont accélérés, focalisés et défléchis à l'aide d'un jeu de plaques de déflexion. La variation des tensions appliquées sur les deux plaques permet d'obtenir un balayage de l'image électronique de la fente sur l'écran électroluminescent de sortie. Cet écran convertit les électrons en photons qui vont alors impressionner le film photographique (TRI-X ou TMAX-400 de chez Kodak) en contact avec l'écran électroluminescent.

Le dispositif d'acquisition et de traitement se compose d'un scanner TRUVEL TF-3X (résolution 720DPI, 1 pixel $=35 \mu \mathrm{m} \times 35 \mu \mathrm{m}$ ), d'un PC et d'un ensemble de logiciels (Image-In, Excel, Matlab). 


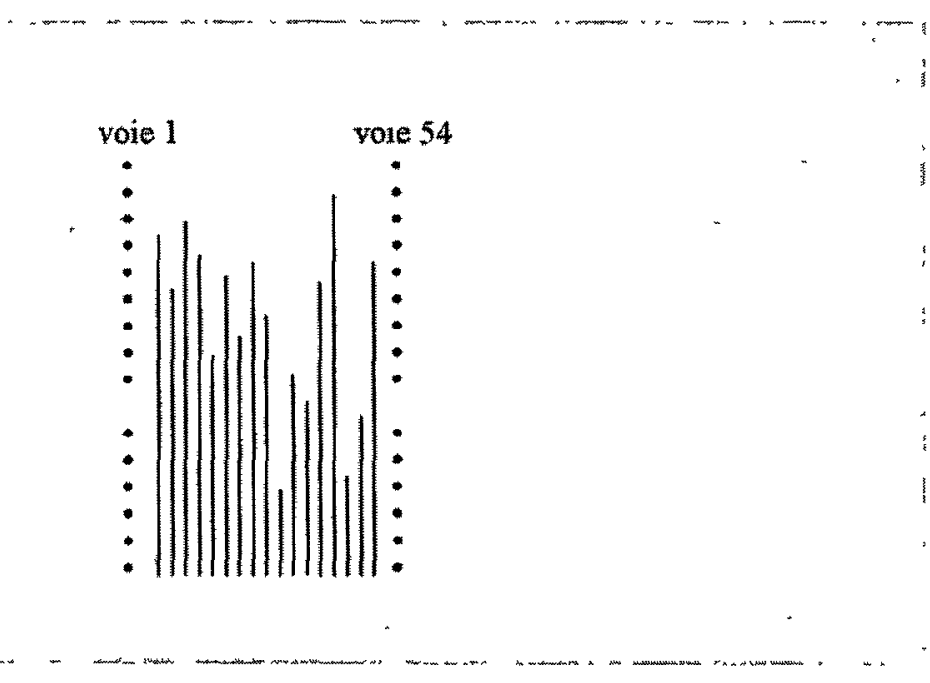

Fig. 2 - Un exemple de film

[An example of a film ]

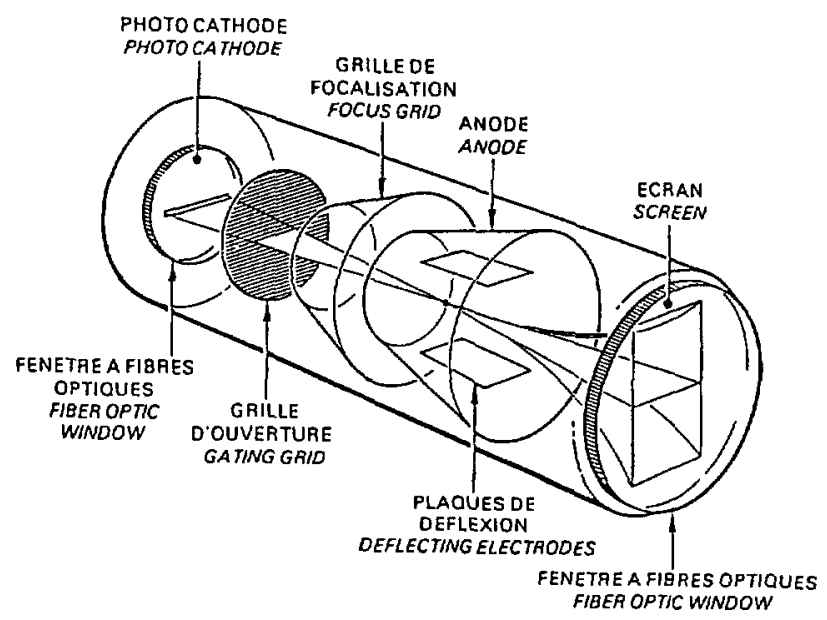

Fig 3. - Le tube convertisseur d'mages

[The streak camera tube.]

2.3. Principe de la Chronométrie. - Puisque la caméra sert de chronomètre, une information temporelle est associée à chaque point de l'écran électroluminescent. Actuellement, pendant les expérimentations, les deux bases temporelles synchrones permettent de restituer la chronologie des événements étudiés.

L'exploitation classique des clichés repose sur l'hypothèse que l'image de la fente obtenue sur le film est une droite (Fig. 4). Elle passe par les deux marqueurs synchrones fournis par le générateur d'impulsions. 


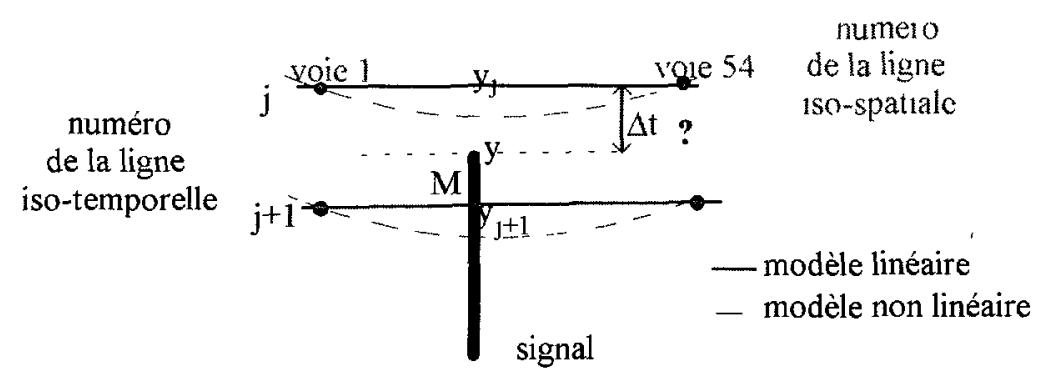

Fıg. 4. - Principe de la chronométrie.

[Prınciple of the temporal calibration.]

Dans ces conditions et pour le cas où la vitesse de balayage est constante, l'instant d'occurrence d'un événement en un point $M$ du cliché est obtenu à partir de :

$$
t_{y}=t_{y_{2}}+\frac{y-y_{3}}{y_{\jmath}+1-y_{\jmath}}\left(t_{y_{\jmath}+1}-t_{y_{\jmath}}\right)
$$

Si les lignes iso-temporelles ne sont pas des droites, hypothèse que nous allons vérifier, les coordonnées $y_{3}$ et $y_{j+1}$ sont modifiées et donc l'instant $t_{y}$. Une connaissance précise du cadre spatio-temporel est donc nécessaire, et doit ainsi permettre de déterminer pour chaque point de l'écran de sortie de la caméra, un temps avec la plus grande exactitude.

Par ailleurs, la vitesse de balayage de la caméra, liée aux tensions de déflexion du tube convertisseur d'images, est en principe constante. Il apparaît intéressant d'étudier l'évolution de cette vitesse pendant la durée du balayage. Si des fluctuations de vitesse (accidentelles ou non) existent, elles devront être prises en compte dans le calcul de l'instant d'occurrence.

\section{Modélisation}

3.1. ACQUISITION DES DONNÉES. - L'approche réalisée ici consiste à construire un modèle vérifiant les conditions pratiques de fonctionnement du récepteur, constitué de la caméra, de son film et d'un scanner (TRUVEL TF-3X). Pour cela on utilise une approche par modélisation du cadre spatio-temporel généré par le récepteur [5]. Elle doit mettre en évidence les défauts géométriques du système et les intégrer dans l'exploitation des clichés.

À cette fin, les deux signaux synchrones servant de base de temps sont repris par deux coupleurs optiques (une entrée - huit sorties par coupleur). Nous disposons ainsi de 16 voles optiques synchrones alignées sur la photocathode du tube convertisseur d'images (Fig. 5). Le film ainsi obtenu (Fig. 6) est numérisé à l'aide du scanner TRUVEL.

La première étape consiste alors à extraire les coordonnées $X$ et $Y$ représentatives du temps associé à chacune des impulsions observées sur le film. Pour cela, nous avons développé un logiciel faisant appel à des techniques de traitement d'images (utilisation conjointe de méthodes de seuillage et de masques de Prewitt). 


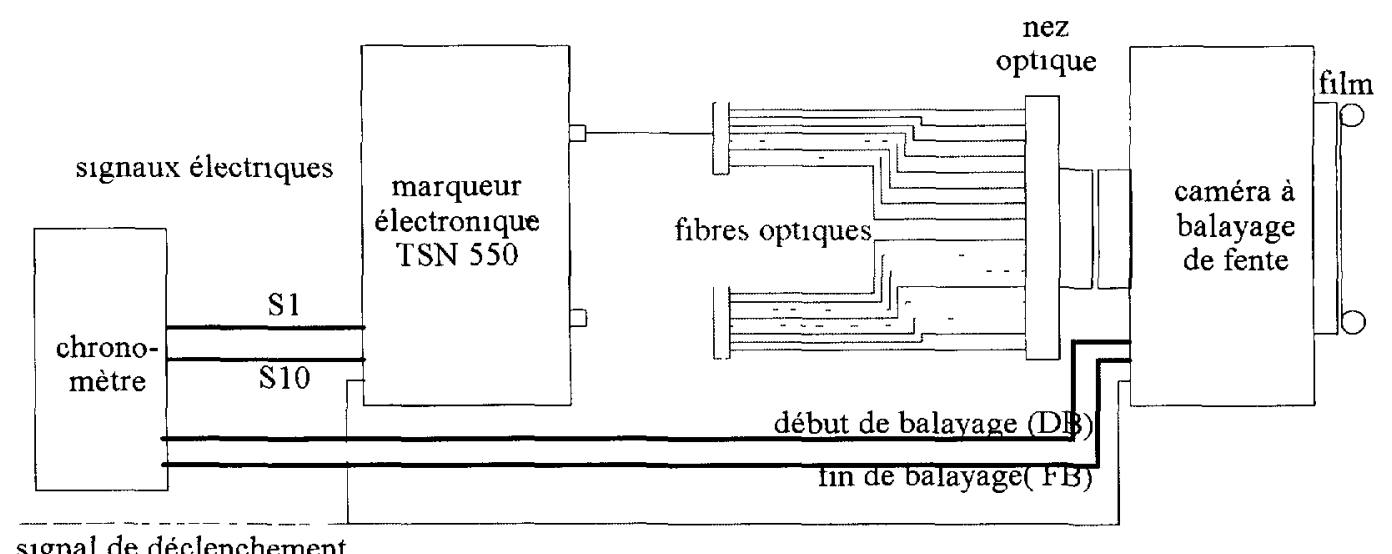

signal de déclenchement

Fig. 5. - Banc expérimental.

[Experimental arrangement.]

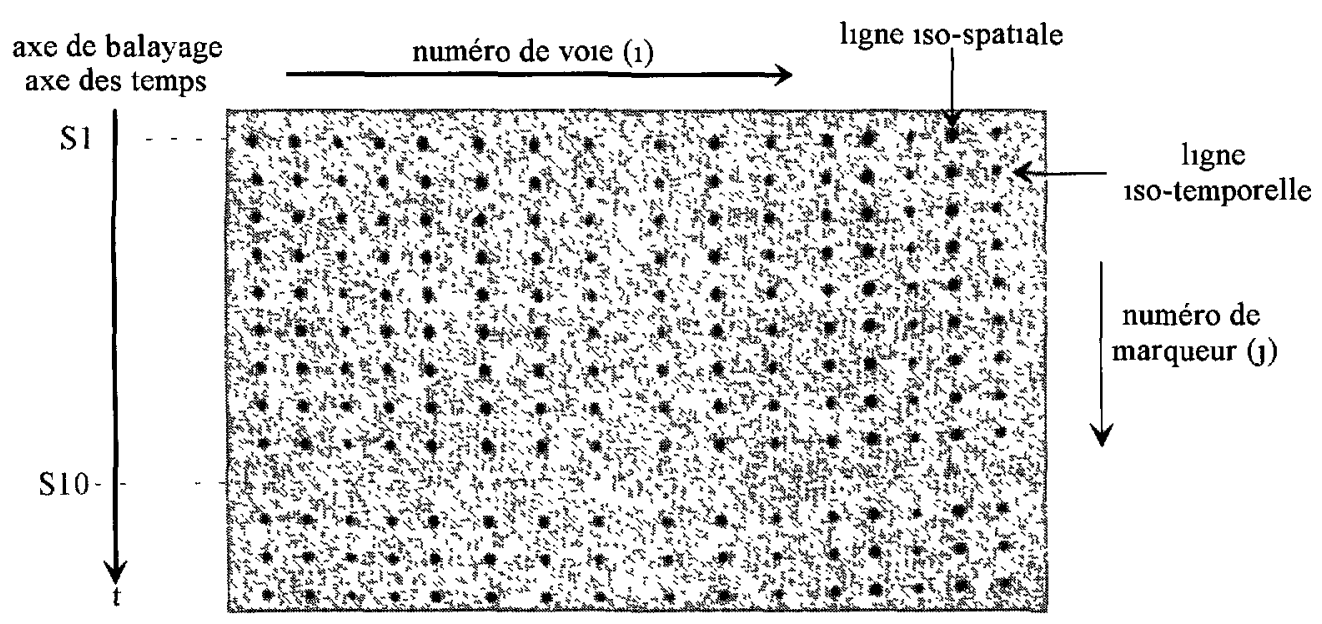

Fig 6. - Partıe d'un film servant à la modélısation

[Part of a film using for the modelisation ]

Le choix de la référence temporelle sur l'image de l'impulsion lumineuse obtenue sur le film est primordial. En effet sa taille varie en fonction de plusieurs paramètres :

- qualité du couplage entre la fibre optique et la photocathode du tube (au niveau du nez adaptateur) ;

- puissance du signal lumineux se propageant dans la fibre, et donc de l'intensité du faisceau d'électrons dans le tube convertisseur d'images de la caméra ;

- défocalisation de ce faisceau d'électrons (minimale au centre de l'écran de sortie).

La référence temporelle retenue pour chacune des images des impulsions lumineuses observées sur le cliché est leur barycentre. En effet, la répartition spatiale de l'énergie de l'impulsion 


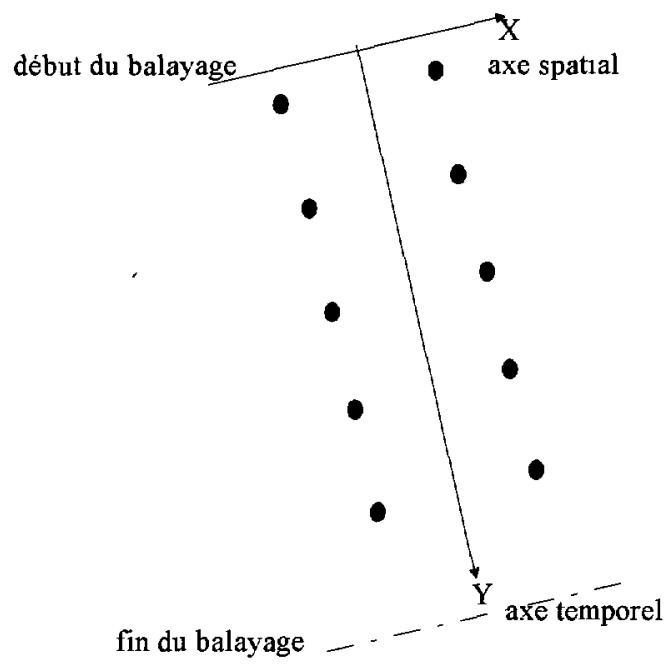

Fıg. 7. - Positionnement du repère.

[Position of spatial axıs and temporal axis ]

lumineuse peut être considérée comme gaussienne [6], le maximum d'énergie peut donc être associé à son barycentre. Ce choix permet de recaler la chronométrie électrique du générateur TSN 550 avec les bases de temps sur le film indépendamment des effets cités ci-dessus.

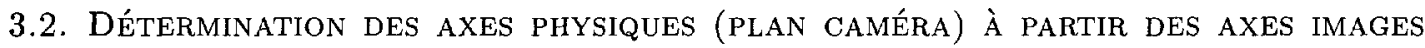
(PLAN FILM). - En nous plaçant immédiatement dans le cadre de l'interprétation des images des caméras à balayage de fente, toutes les mesures effectuées font intervenir deux grandeurs : l'une d'espace (selon l'axe de la fente) et l'autre de temps (selon l'axe des temps). C'est pourquoi le préalable à toute modélisation (et à toute analyse des signaux) est la recherche de ces deux axes "physiques" de la caméra à partir des directions principales du repère "image". Ceci doit permettre d'aboutir à un modèle par caméra, indépendamment de la durée de balayage.

Le choix de l'axe temporel sur le film se fait aisément. Par convention, cet axe est positionné selon une direction principale entre les voies 27 et 28 . Le choix de l'axe de la fente est plus délicat. L'axe spatial correspond à l'image de la fente à $t=0$, c'est-à-dire à la position de début de balayage. Cet axe est perpendiculaire à l'axe temporel (Fig. 7).

Les positions de début de balayage et de fin de balayage sont des informations absentes en pratique. Pour estimer ces positions, nous définissons un modèle de "trajectoire" pour les marqueurs d'une ligne iso-spatiale. Soit $\mathrm{M}$ le marqueur $k$ ( $k$-ième impulsion) de la voie $i$, de coordonnées $\left(X_{\imath k}, Y_{\imath k}\right)$ dans le plan du film (ou plan image), chacun de ces points permet de définir une trajectoire modélisée en fonction du temps par un polynôme donné par la relation (2). On fait alors l'hypothèse que le balayage de la caméra peut être non linéaire, ce qui entraîne des variations de vitesse de balayage, donc des modifications de l'accélération. Pour simplifier, nous ne présentons l'étude que pour une seule coordonnée désignée $z_{k}$ ( $k$ étant un entier, correspondant au numéro du marqueur)

$$
z_{k+1}=z_{k}+\dot{z}_{k} t+\frac{1}{2} \ddot{z}_{k} t^{2}
$$


L'évolution de la "trajectoire" est donc décrite par la représentation d'état :

$$
\underline{Z}_{k+1}=\Phi_{k+1} \underline{Z}_{k}
$$

et observée par (4) :

$$
z_{k+1}=H \underline{Z}_{k+1}
$$

avec :

$$
\underline{Z}_{k}=\left(\begin{array}{l}
z_{k}=\text { position du marqueur } \\
\dot{z}_{k}=\text { vitesse de balayage } \\
\ddot{z}_{k}=\text { accélération de balayage }
\end{array}\right)
$$

$\Phi_{k}$ : la matrice de transition définissant le modèle de trajectoire, qui dépend de la coordonnée de travail $(X$ ou $Y), H_{k}=H=\left(\begin{array}{lll}1 & 0 & 0\end{array}\right)$ : la matrice d'observation.

Pour la recherche de la position du début et de la fin du balayage, on définit la matrice de transition ainsi :

$$
\begin{aligned}
\Phi_{k}=\left(\begin{array}{ccc}
1 & 1 & 1 / 2 \\
0 & 1 & 1 \\
0 & 0 & 1
\end{array}\right) & (\text { pour } X) \\
\Phi_{k}=\left(\begin{array}{ccc}
1 & p & 1 / 2 p^{2} \\
0 & 1 & p \\
0 & 0 & 1
\end{array}\right) & (\text { pour } Y)
\end{aligned}
$$

avec $p$ représentant soit :

- le pas inter-marqueur en microseconde, quand la positıon suivante correspond à un marqueur présent ;

- deux pas inter-marqueurs, quand la position suivante correspond à un marqueur absent ;

- la différence de temps entre le début de balayage et le premier marqueur visible ;

- la différence de temps entre le dernier marqueur visible et la fin du balayage.

Nous définissons l'erreur résiduelle de sortie $\epsilon_{k}$ comme la différence entre la mesure réelle $z_{k}$ de la position du marqueur (le barycentre) déterminée par le programme développé à cet effet, et la mesure estimée par le modèle $\hat{z}_{k}$ :

$$
\epsilon_{k}=z(\text { réel })_{k}-\hat{z}_{k}
$$

Des algorithmes d'estimations récursives $[7,8]$ permettent d'estimer la "trajectoire" en tenant compte des non-stationnarités (vitesses de balayage non constantes). Ces algorithmes sont basés sur les moindres carrés récursifs avec facteur d'oubli. On estime ainsi l'évolution de la vitesse de balayage, de l'accélération, puis les positions de début et de fin de balayage L'algorithme est décrit ainsi [9] :

1) mise à jour de la matrice de covariance $P$ et de la matrice gain $K$ :

$$
\begin{aligned}
& P_{k / k-1}=\Phi_{k} P_{k-1 / k-1} \Phi_{k}^{\mathrm{T}} \\
& K_{k}=P_{k / k-1} H^{\mathrm{T}}\left(H P_{k / k-1} H^{\mathrm{T}}+\lambda\right)^{-1} \\
& P_{k / k}=\frac{P_{k / k-1}-K_{k} H P_{k / k-1}}{\lambda}
\end{aligned}
$$

2) estimation du vecteur d'état à l'échantillon $k$ :

$$
\underline{\hat{Z}}_{k}=\underline{\hat{Z}}_{k / k-1}+K_{k}\left(\hat{z}_{k}-H \underline{\underline{Z}}_{k / k-1}\right)
$$


3) prédiction du vecteur d'état à l'échantillon $k+1$ :

$$
\underline{\hat{Z}}_{k+1 / k}=\Phi_{k+1} \underline{\hat{Z}}_{k}
$$

4) prédiction de la position à l'échantillon $k+1$ :

$$
\hat{z}_{k+1 / k}=H \Phi_{k+1} \underline{\hat{Z}}_{k} .
$$

Le déroulement consiste à estimer le vecteur d'état $Z_{k}$ à partir des observations passées $z$ (réel) ${ }_{\imath}$ $(\imath \leq k)$. On réalise ensuite la prédiction de la position $z_{k+1}$. On peut ansi prédire les positions de fin et de début de balayage, par un algorithme "avant" et "arrière". Les matrices de transition sont différentes suivant que l'on cherche à prédire la position des marqueurs ou la position de début et de fin de balayage. L'algorithme, le gain $K$ et la matrice de variance covariance convergent, assurant ainsi la robustesse. Le vecteur gain $K$ représente la pondération de l'erreur pour l'estimation de la position, de la vitesse et de l'accélération à un instant donné. On va pouvoir ainsi prédıre les positions des marqueurs avec une bonne précision (inférieure au pixel, $35 \mu \mathrm{m} \times 35 \mu \mathrm{m})$.

Le facteur d'oubli $\lambda$ est fixé en général entre 0,3 et 1 . Il donne des caractéristiques générales de poursuite au système (lissage du bruit ou adaptation aux ruptures). Après différents tests, nous le fixons à 0,7 , ce qui permet de lisser suffisamment le bruit de mesure.

Pour la recherche du début du balayage, nous l'initialisons avec le dernier marqueur. La vitesse initiale est le rapport de la taille de la fenêtre $(50 \mathrm{~mm})$ sur la durée L'accélération initiale est prise légèrement différente de zéro (vitesse non constante). La même démarche est faite en sens inverse pour la position de la fin du balayage.

La position du début et de la fin de balayage rend accessible une autre grandeur, à savoir la taille de la fenêtre d'observation réelle, à comparer avec la taille théorique $(50 \mathrm{~mm})$.

Le changement de repère entre le plan film et le plan caméra nécessite une opération de rotation et de translation classique. Nous pouvons maintenant modéliser le cadre spatio-temporel généré par la caméra.

3 3. Modélisațion GÉométrique. - Classiquement, les images obtenues avec des caméras peuvent être considérées comme des surfaces. Pour avoir une représentation de ces dernières, on peut réaliser une modélisation géométrique [10]. Pour les caméras à balayage de fente, l'image contient une dimension temporelle. La surface se limite donc à l'ımage de la fente à un instant donné, c'est-à-dire à une ligne iso-temporelle. Avec le dispositif expérimental de la figure 5, nous disposons de 16 points pour reconstruıre l'image de la fente à un instant donné.

La réalisation d'une opération de filtrage polynomial, sur les données transposées dans le plan caméra, permet de construire un jeu de courbes 1so-temporelles s'ajustant au mieux à l'ensemble des mesures expérimentales au sens du critère des moindres carrés (Fig. 8). L'étude du cadre spatio-temporel montre alors que les lignes iso-temporelles ne sont pas des droites mais des paraboles. Le modèle du cadre spatio-temporel peut donc s'écrire :

$$
y_{\imath \jmath}=a_{\jmath} x_{\imath \jmath}^{2}+b_{\jmath} x_{\imath \jmath}+c_{\jmath}
$$

avec $\imath$ : numéro de la voie et donc de la ligne iso-spatiale , $\jmath$ : numéro du marqueur et donc de la ligne iso-temporelle.

Les différentes lignes iso-temporelles correspondent aux différentes images estimées de la fente obtenues aux instants considérés. De plus il peut exister des fluctuations de tension sur les plaques de déflexion. Ceci entraîne des sauts de vitesse qu'il faut prendre en compte pour modéliser l'évolution des lignes iso-temporelles. La variable $y_{0 j}$ est le point d'intersection entre 


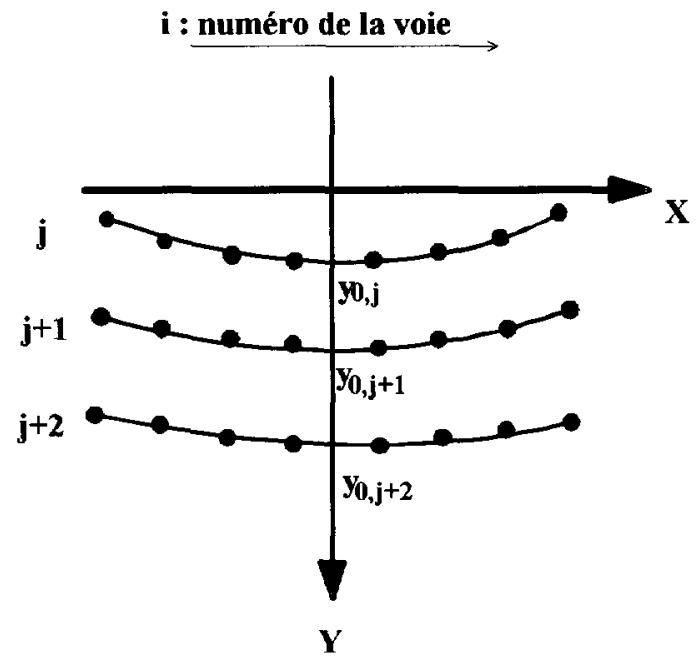

Fig. 8. - Lignes iso-temporelles.

[Iso-temporal lines ]

la ligne iso-temporelle et l'axe temporel $Y$ (Fig. 8). Elle permet de prendre en compte les sauts de tension, donc les sauts de vitesse de balayage.

On constate que les coefficients estimés $a_{\jmath}, b_{\jmath}, c_{\jmath}$ de chaque ligne iso-temporelle évoluent, en raison notamment de la distance parcourue par les électrons dans le tube convertisseur d'images qui est différente selon qu'on se situe en début, au milieu ou en fin de balayage. Les lois d'évolution des coefficients $a_{\jmath}, b_{j}, c_{\jmath}$ en fonction de la variable $y_{0}$ ont été déterminées empiriquement :

$$
\left\{\begin{array}{l}
a_{3}=\alpha_{0}+\alpha_{1} y_{0} \\
b_{3}=\beta_{0}+\beta_{1} y_{0 \jmath} \\
c_{\jmath}=\gamma_{1} y_{0 \jmath}
\end{array}\right.
$$

En exprimant $a_{\jmath}, b_{\jmath}, c_{\jmath}$ en fonction de $\alpha_{0}, \alpha_{1}, \beta_{0}, \beta_{1}, \gamma_{1}$ et de $y_{0}$ dans l'équation (6), on obtient :

$$
y_{\imath \jmath}=\alpha_{0} x_{\imath \jmath}^{2}+\alpha_{1} y_{0 \jmath} x_{\imath \jmath}^{2}+\beta_{0} x_{\imath \jmath}+\beta_{1} y_{0 \jmath} x_{\imath \jmath}+y_{0 \jmath} .
$$

L'équation (8) donne la relation entre l'ordonnée du marqueur $y_{\imath \jmath}$ via $y_{0 \jmath}$, et son abscisse $x_{\imath \jmath}$ dans le "plan caméra". Chaque position de marqueur est solution d'une équation. Cet ensemble d'équations peut s'écrire sous forme matricielle :

$$
\underline{Y}=\alpha_{0} \underline{X_{1}}+\alpha_{1} \underline{X_{2}}+\beta_{0} \underline{X_{3}}+\beta_{1} \underline{X_{4}}+\gamma_{1} \underline{X_{5}}=X \underline{\theta}
$$

avec :

$$
\underline{Y}=\left[\begin{array}{c}
\cdots \\
y_{\imath \jmath} \\
\cdots
\end{array}\right], \underline{X_{1}}=\left[\begin{array}{c}
\cdots \\
x_{\imath \jmath}^{2} \\
\cdots
\end{array}\right], \underline{X_{2}}=\left[\begin{array}{c}
\cdots \\
y_{0 \jmath} x_{\imath \jmath}^{2} \\
\cdots
\end{array}\right], \underline{X_{3}}=\left[\begin{array}{c}
\cdots \\
x_{\imath \jmath} \\
\cdots
\end{array}\right], \underline{X_{4}}=\left[\begin{array}{c}
\cdots \\
y_{0 \jmath} x_{\imath \jmath} \\
\cdots
\end{array}\right], \underline{X_{5}}=\left[\begin{array}{c}
\cdots \\
y_{0 \jmath} \\
\cdots
\end{array}\right]
$$

et avec :

$$
\begin{aligned}
& X=\left[\begin{array}{lllll}
\underline{X_{1}} & \underline{X_{2}} & \underline{X_{3}} & \underline{X_{4}} & \underline{X_{5}}
\end{array}\right] \\
& \underline{\theta^{\mathrm{T}}}=\left[\begin{array}{lllll}
\alpha_{0} & \alpha_{1} & \beta_{0} & \beta_{1} & \gamma_{1}
\end{array}\right] .
\end{aligned}
$$


Tableau I. - Résultat de l'identification.

[Result of the identification.]

\begin{tabular}{|l|c|c|c|c|c|}
\hline paramètres & $\alpha_{0}\left(\mu \mathrm{m}^{-1}\right)$ & $\alpha_{1}\left(\mu \mathrm{m}^{-2}\right)$ & $\beta_{0}$ & $\beta_{1}\left(\mu \mathrm{m}^{-1}\right)$ & $\gamma_{1}\left(\mu \mathrm{m}^{-1}\right)$ \\
\hline modèle 1 & $-2,09 \times 10^{-6}$ & $5,78 \times 10^{-11}$ & $-2,86 \times 10^{-3}$ & $1,58 \times 10^{-7}$ & 1,00 \\
\hline écart type estımé & $0,02 \times 10^{-6}$ & $0,06 \times 10^{-11}$ & $0,13 \times 10^{-3}$ & $0,04 \times 10^{-7}$ & $2 \times 10^{-5}$ \\
\hline modèle 2 & $-2,12 \times 10^{-6}$ & $5,71 \times 10^{-11}$ & $-0,18 \times 10^{-3}$ & $1,79 \times 10^{-7}$ & 1,00 \\
\hline écart type estımé & $0,02 \times 10^{-6}$ & $0,07 \times 10^{-11}$ & $0,14 \times 10^{-3}$ & $0,05 \times 10^{-7}$ & $2 \times 10^{-5}$ \\
\hline
\end{tabular}

Les paramètres sont estimés au moyen de la méthode des Moindres Carrés Simples (MCS) [5]. L'estimateur est donné par la relation :

$$
\underline{\hat{\theta}}=\left(X^{\mathrm{T}} X\right)^{-1} X^{\mathrm{T}} \underline{Y} .
$$

Ce modèle fournit donc un jeu de paramètres liés aux caractéristiques physiques de la caméra. Une étude plus approfondie, portant sur un ensemble de quatorze caméras, nous a permis de vérifier, que les paramètres $\alpha_{0}$ et $\alpha_{1}$ peuvent être considérés comme constants pour une même caméra, quelques soient les conditions expérimentales, notamment, lorsqu'on fait varier la durée de balayage. On peut donc supposer que ces paramètres $\alpha_{0}$ et $\alpha_{1}$, représentatifs du fonctionnement intrinsèque de la caméra, dépendent de ses caractéristiques propres (concavité de la photocathode, distance parcourue par les électrons dans le tube, courbure de l'écran électroluminescent). Par contre, les paramètres $\beta_{0}$ et $\beta_{1}$ sont modifiés pour une même caméra, lorsque l'on déplace l'orientation du nez adaptateur par rapport à la photocathode du tube. Ils prennent donc en compte l'orientation de la fente formée par le nez adaptateur relativement à l'axe d'entrée du tube de la caméra. Le paramètre $\gamma_{1}$ est lié aux tensions appliquées sur les plaques de déflexion.

Le tableau I donne le résultat de l'identification de la régression linéaire pour deux films réalisés avec la même caméra. On peut remarquer d'une part la confiance accordée à l'estimation des paramètres $\alpha_{0}, \alpha_{1}$ et $\gamma_{1}$, d'autre part la fluctuation effective des paramètres $b_{0}$ et $b_{1}$. Cette fluctuation est attribuée au changement de la position du nez adaptateur par rapport à l'axe de balayage.

Les résidus $\epsilon_{k}$ obtenus avec le modèle parabolique sont blancs (moyenne nulle, non corrélés) (Fig. 9).

La figure 10 représente les résidus obtenus avec le modèle dit linéaire (les lignes iso-temporelles sont des droites) pour le même film que celui utilisé pour la figure 9 . Les résidus sont à comparer au poids du pixel, qui est ici de 11 ns pour une durée de balayage de $15 \mu \mathrm{s}$.

Ces différentes expériences ont été renouvelées sur neuf autres caméras. Pour chacune d'entre elles, nous avons obtenu des résultats comparables. Chaque modèle obtenu pour chaque caméra permet d'améliorer sensiblement l'exactitude de la mesure.

\section{Validation des performances obtenues avec le modèle parabolique}

4.1. Nombre optimal de voies de marqueurs. - Il s'agit de déterminer, ici, le nombre de voies de marqueurs (ou bases temporelles) nécessaire, pour obtenir un modèle représentatif du cadre spatio-temporel de la caméra, compte tenu de la précision recherchée sur l'échelle temporelle. Il faut trouver un compromis, car chaque voie utilisée comme base temporelle entraîne en pratique trois voies de mesure en moins (les deux voies adjacentes). Nous avons donc calculé les paramètres du modèle spatio-temporel obtenu en utilisant successivement 


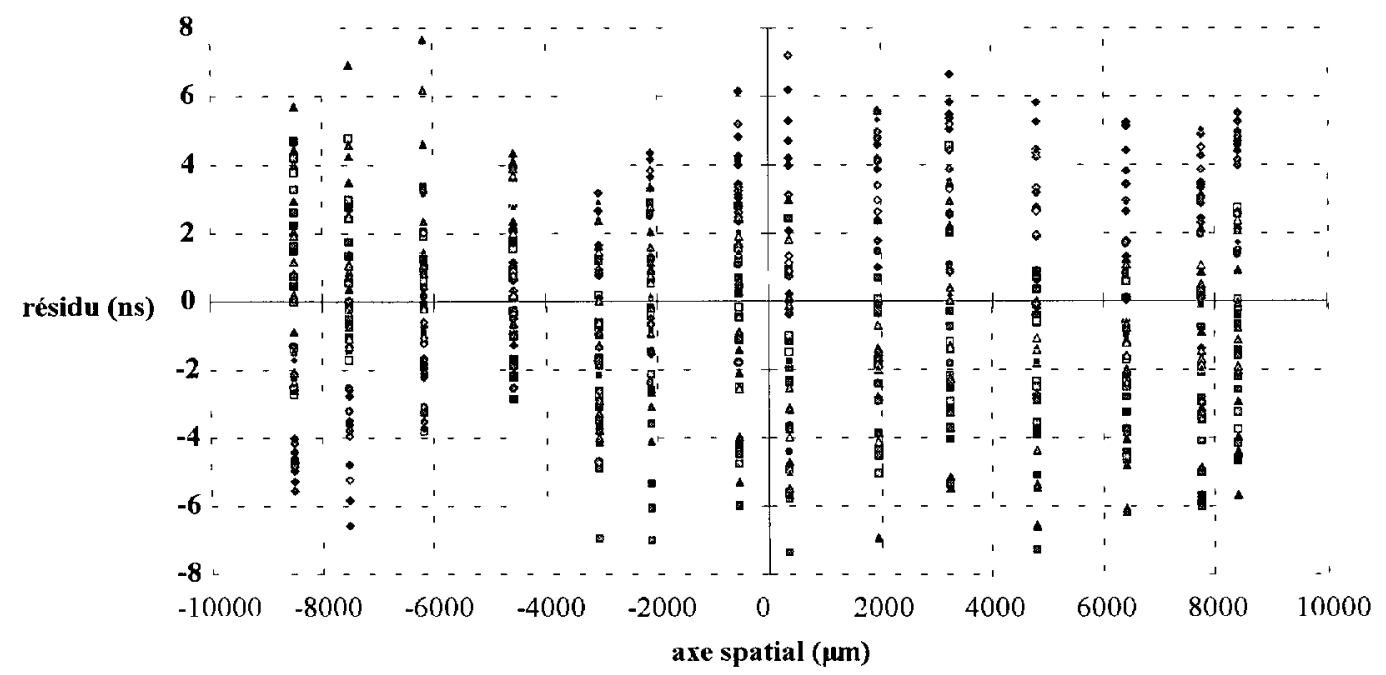

Fig 9 - Résıdus avec le modèle parabolique pour une durée de balayage de $15 \mu \mathrm{s}$.

[Resıdual errors with a parabolic model for a duration of scanning of $15 \mu \mathrm{s}$.]

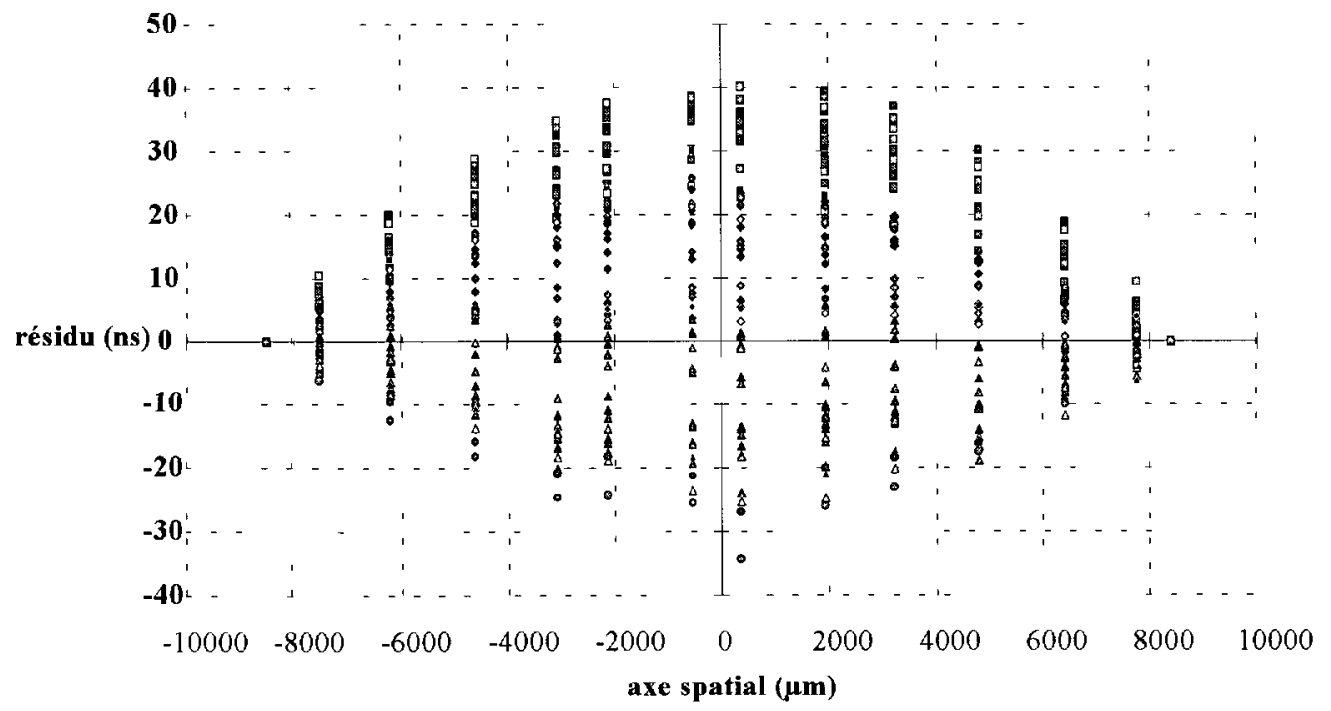

Fıg. 10. - Résıdus avec le modèle dit lınéaire pour une durée de balayage de $15 \mu \mathrm{s}$.

[Residual errors with a linear model for a duration of scannıng of $15 \mu \mathrm{s}$.]

4, 6,8 et 16 voies de marqueurs. Nous comparons la précısion de ces modèles avec celle obtenue à l'aide de l'approche linéaire classique prenant en compte deux voies seulement (modèle des droites).

Les résultats de la figure 11 sont ceux obtenus pour une durée de balayage de $15 \mu \mathrm{s}$. Nous remarquons que l'écart-type du résidu est équivalent, que le modèle soit calculé avec 16, 8,6 ou 4 voies de marqueurs. Le test de normalité (drolte de Henry [11]) montre que la distribution 


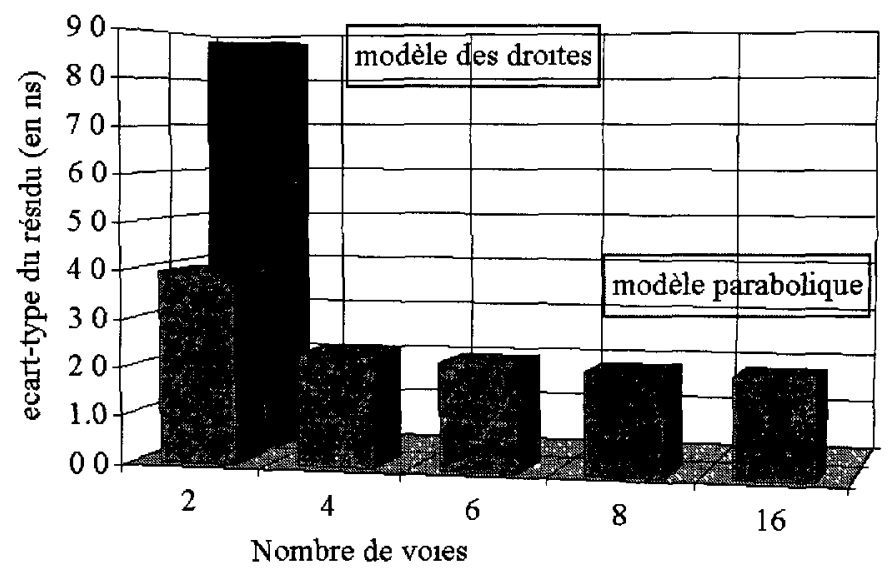

Fig 11. - Écart-type expérimental du résidu en fonction du nombre de voles

[Standard deviation of the residu versus the number of channels.]

des résidus est gaussienne. La précision ainsi obtenue est donc équivalente sur toute la surface d'enregistrement du film.

La gamme des durées de balayage étudiées couvre le domaine $1 \mu \mathrm{s}-20 \mu \mathrm{s}$. Les résultats exprimés en $\mu \mathrm{m}$ sont équivalents quelle que soit cette durée d'observation. L'écart-type expérimental du résidu est d'environ $8 \mu \mathrm{m}$. On précise qu'un pixel représente $35 \mu \mathrm{m} \times 35 \mu \mathrm{m}$ sur le film.

Nous avons comparé les paramètres des modèles calculés avec 8,6 et 4 voies avec les paramètres du modèle calculés avec 16 voies. La statistique fournie par la loi de Student montre que les modèles peuvent être considérés comme identiques entre eux avec une probabilité de $95 \%$ Ainsi, quatre bases temporelles suffisent pour obtenir un modèle représentatif de la géométrie du cadre spatio-temporel généré par la caméra à balayage de fente. Les résidus calculés avec un tel modèle sur un film comportant 16 voies de marqueurs restent inférieurs au pixel dans tous les cas (pour une durée de balayage pouvant évoluer de $1 \mu$ s à $20 \mu \mathrm{s}$ ).

Dans l'approximation gaussienne, cas étudié, la probabilité pour que le résidu (écart entre la position mesurée et celle estimée par le modèle) soit supérieur au pixel est de $20 \%$ pour le modèle basé sur les droites iso-temporelles (1,282 écart-type $>35 \mu \mathrm{m}$, avec un écart-type de $28 \mu \mathrm{m})$. Cette même probabilité est d'environ $0,01 \%$ pour le modèle basé sur les paraboles iso- temporelles (avec 4 voies).

4.2. VÉRIFICATION EXPÉRIMENTALE. - La validation de ce modèle et son incidence sur l'exactitude des mesures peuvent être vérifiées à l'aide du montage décrit en figure 12 . Un générateur d'impulsions ( $\mathrm{n}^{\circ} 3$ ) fournit une série d'impulsions dont on connaît parfaitement les caractéristiques temporelles (grandeurs conventionnellement vraies). Ces impulsions alimentent en même temps deux caméras ; leurs traces optiques vont donc apparaître au même instant sur les deux clichés. La durée de balayage est de $15 \mu \mathrm{s}$. Le poids d'un pixel est de $11 \mathrm{~ns}$.

Le tableau II montre les écarts entre le temps donné par le chronomètre et le temps calculé sur le film avec le modèle dit linéaire et avec le modèle parabolique. Ces résultats sont à comparer à la précision du chronomètre qui est de 1 ns.

En modélisant le cadre spatio-temporel de la caméra avec quatre voies de marqueurs au moins, nous sommes donc en mesure de garantir les résultats avec une précision meilleure que le poids du pixel. Cette précision est indépendante de la position du signal sur le film. 


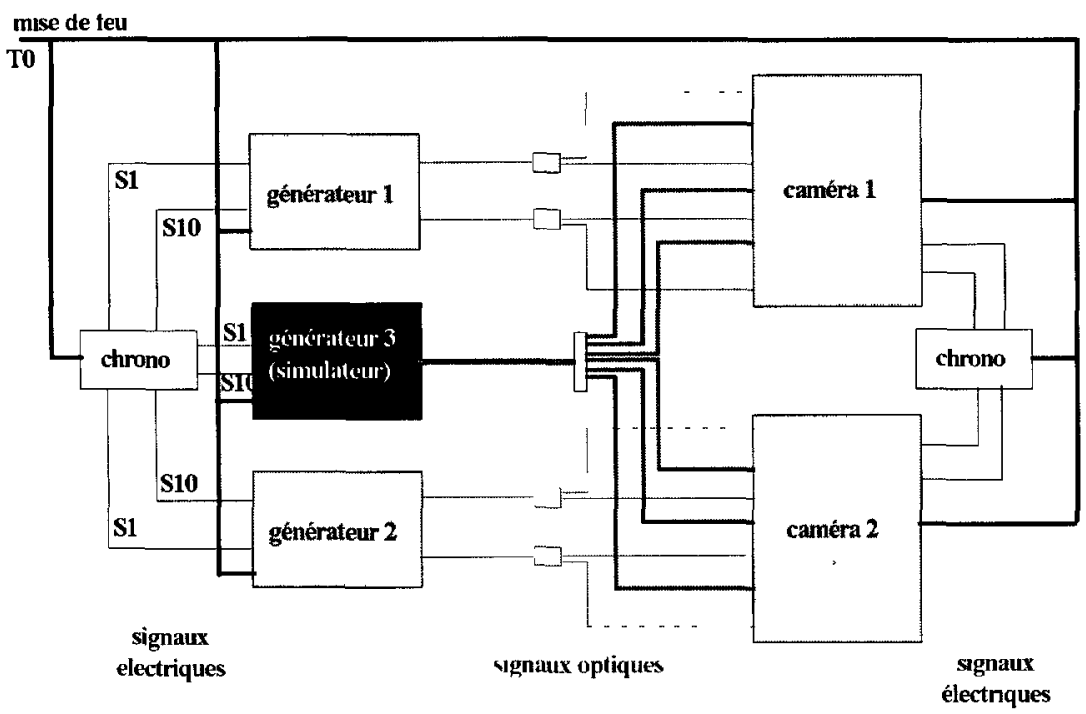

Fıg. 12 - Dispositif expérimental

[Experimental set-up.]

Tableau II. - Les résultats de la sımulatıon.

[Results of the simulation.]

\begin{tabular}{|c|c|c|}
\hline & $\begin{array}{c}\text { approche } \\
\text { linéaire }\end{array}$ & $\begin{array}{c}\text { approche } \\
\text { parabolique } \\
\text { (4 voies) }\end{array}$ \\
\hline $\begin{array}{c}\text { écart observé entre le chronomètre } \\
\text { (référence temporelle) et une caméra } \\
\text { (temps estimé) (ns) }\end{array}$ & -36 à 16 & \pm 4 \\
\hline $\begin{array}{c}\text { écart observé entre les deux caméras } \\
\text { (temps estimés) (ns) }\end{array}$ & -16 à 56 & \pm 4 \\
\hline
\end{tabular}

\section{Conclusion}

Les caméras à balayage de fente sont utilisées pour observer des phénomènes rapides. Cette étude a permis d'améliorer la qualité de la mesure, grâce à la connaissance d'un modèle du cadre spatio-temporel généré par la caméra à balayage de fente. L'approche proposée consiste à modêliser ce cadre en réalisant une opération de filtrage polynomial.

Le modèle parabolique fournit un jeu de paramètres fonction des caractéristiques physiques de la caméra. Par conséquent, un tel modèle peut être utilisé pour diagnostiquer des modifications éventuelles des performances des caméras au cours de leur utilisation.

Pour utiliser un tel modèle, il est nécessaire de disposer de quatre bases temporelles, qui se sont avérées suffisantes pour caractériser complètement le cadre spatio-temporel généré par la caméra. Les performances chronométriques obtenues avec ce modèle sont remarquables et équivalentes pour toutes les caméras étudiées (dix au total). En effet, pour une durée de 
balayage de $15 \mu \mathrm{s}$, par exemple, l'exactitude de la mesure estimée avec un tel modèle est de 4 ns, sur des signaux impulsionnels, alors que le poids du pixel est de 11 ns. De plus, la qualité de la mesure ne dépend pas de la position du point de mesure sur le film.

Par ailleurs, la démarche utilisée ici peut être appliquée à tout autre système fonctionnant sur le principe d'un tube à balayage, pourvu que l'on dispose d'un signal de référence suffisamment précis et d'un dispositif de numérisation de l'image.

\section{Bibliographie}

[1] Kuffel J., Malewski R. and van Heeswijk R.G., Modeling of the dynamic performance of transient recorders used for high voltage impulse tests, IEEE Trans. on Power Delivery 6 (1991) 507-515.

[2] Wilson S.K. and Delay P., A method to improve cathode ray oscilloscope accurancy, IEEE Trans. Instrum. Measurement 43 (June 1994).

[3] Gauthier J.P. et Bouchu M., Mesure en hydrodynamique au moyen de sondes optiques et d'un enregistrement par caméra électronique à fente, 13th International Congress on High Speed Cinematography (Tokyo, 1978).

[4] Notice caméra TSN 506 N. Thomson CSF. Not/1019 - 11/87.

[5] Oeconomos J.N., Garcin G. et Ansart J.P., Analyse fréquentielle d'images d'interférométrie Doppler laser, Quatorzième colloque GRETSI (Juan les Pins, du 13 au 16 septembre 1993) pp. $723-726$.

[6] Brunet J., Jaume D., Labarrère M., Rault A. et Vergé M., Détection et diagnostic de pannes. Approche par modélisation (Ed. Hermes, Paris, 1990).

[7] D'Humières E., Bernet J.M. and Imhoff C., Single point streak tube, SPIE Vol. 648 Photonics: High Bandwidth Analog Applications (1986).

[8] Berniere A., Bette G. and D'Apuzzo M., Movement Problems Arising from the Use of the Recursive Algorithm for Model Identification of Electrical Systems, IEEE. Trans. in Instrumentation and measurement 41 (April 1992).

[9] Leger J.M., Brochard J. et Leard M., Détection de rupture de trajectoire en poursuite de cible, Colloque "Automatique, Génie informatique, Image (Poitiers - Futuroscope, 2 et 3 juin 1994).

[10] Gardan Y., Mathématiques et CAO. Méthodes de base (Ed. Hermès, Paris, 1985).

[11] Statıstique appliquée à l'exploitation des mesures. CEA. Tome 1 et 2. Ed. Masson (Paris, 1978). 